\title{
Existence and Global Exponential Stability of Periodic Solutions for General Neural Networks with Time-Varying Delays
}

\author{
Xinsong Yang \\ Department of Mathematics, Honghe University, Mengzi, Yunnan 661100, China \\ Correspondence should be addressed to Xinsong Yang, xinsongyang@163.com
}

Received 28 October 2007; Revised 1 February 2008; Accepted 14 April 2008

Recommended by Attila Gilanyi

By using the coincidence degree theorem and differential inequality techniques, sufficient conditions are obtained for the existence and global exponential stability of periodic solutions for general neural networks with time-varying (including bounded and unbounded) delays. Some known results are improved and some new results are obtained. An example is employed to illustrate our feasible results.

Copyright (C) 2008 Xinsong Yang. This is an open access article distributed under the Creative Commons Attribution License, which permits unrestricted use, distribution, and reproduction in any medium, provided the original work is properly cited.

\section{Introduction}

In recent years, the delayed cellular neural networks (DCNNs) have been extensively studied because of their immense potentials of application perspective in different areas such as pattern recognition, optimization, and signal and image processing [1-3]. Hence, they have been the object of intensive analysis by numerous authors, and some interesting results on the existence and stability of periodic and almost periodic solutions have been obtained [4-12]. To our knowledge, few authors have considered global stability of periodic solutions for the neural networks with bounded and unbounded time-varying delays. In theory and application, global stability of periodic solutions of DCNNs is of great importance since the global stability of equilibrium points can be considered as a special case of periodic solution with zero period [8]. Hence, in this paper, we will study the existence and global exponential stability of periodic solutions of the following general neural networks with time-varying delays:

$$
\begin{aligned}
x_{i}^{\prime}(t)=-a_{i}\left(t, x_{i}(t)\right)+\sum_{j=1}^{n} & {\left[a_{i j}(t) f_{j}\left(x_{j}(t)\right)+b_{i j}(t) f_{j}\left(x_{j}\left(t-\tau_{i j}(t)\right)\right)\right.} \\
& \left.+c_{i j}(t) \int_{-\infty}^{t} k_{i j}(t-s) g_{j}\left(x_{j}(s)\right) \mathrm{d} s\right]+I_{i}(t), \quad i=1,2, \ldots, n,
\end{aligned}
$$


where $x_{i}(t)$ is the state of neuron, $i=1,2, \ldots, n, A=\left(a_{i j}\right)_{n \times n}, B=\left(b_{i j}\right)_{n \times n}$, and $C=\left(c_{i j}\right)_{n \times n}$ are connection matrices, $I=\left(I_{1}, I_{2}, \ldots, I_{n}\right)^{T}$ is the input function, and $f(x)=\left(f_{1}\left(x_{1}\right), f_{2}\left(x_{2}\right)\right.$, $\left.\ldots, f_{n}\left(x_{n}\right)\right)^{T}$ is the activation function of the neurons.

DCNNs in [4-12] and the references cited therein are special cases of (1.1). In particular, when $a_{i}\left(t, x_{i}(t)\right)=a_{i} x_{i}(t), a_{i j}(t)=a_{i j}, b_{i j}(t)=b_{i j}, c_{i j}(t)=c_{i j}, I_{i}(t)=I_{i}, a_{i}, a_{i j}, b_{i j}, c_{i j}, I_{i}$ are constants, the authors of [9] considered the existence and global exponential stability for (1.1) with periodic impulses. The methods used in [9] are Mawhin's coincidence degree theorem [13] and Lyapunov functions. In [14], by using Mawhin's coincidence degree theorem [13], the authors investigated the global existence of positive periodic solutions of mutualism systems with bounded and unbounded time-varying delays, and some sufficient conditions are obtained. In [12], the authors considered (1.1) when $a_{i}, a_{i j}, b_{i j}, c_{i j}$ are constants.

We assume what follows.

$\left(\mathrm{H}_{1}\right) a_{i j}(t), b_{i j}(t), c_{i j}(t), \tau_{i j}(t), I_{i}(t)$ are continuous $\omega$-periodic functions, and $a_{i}\left(t, x_{i}(t)\right)$ are continuous $\omega$-periodic in the first variable. $\tau_{i j}(t) \geq 0, i, j=1,2, \ldots, n$.

$\left(\mathrm{H}_{2}\right)$ There exist positive constants $\underline{a}_{i}$ and $\bar{a}_{i}$ such that $\left(a_{i}(t, u)-a_{i}(t, v)\right)(u-v)>0$, and $\underline{a}_{i}|u| \leq\left|a_{i}(t, u)\right| \leq \bar{a}_{i}|u|$, for all $t, u, v \in R, i=1,2, \ldots, n$.

$\left(\mathrm{H}_{3}\right) f_{j}, g_{j} \in C(R, R), j=1,2, \ldots, n$. There are positive constants $L_{j}^{f}>0, L_{j}^{g}>0$, such that $\left|g_{j}(x)-g_{j}(y)\right| \leq L_{j}^{g}|x-y|,\left|f_{j}(x)-f_{j}(y)\right| \leq L_{j}^{f}|x-y|$, for all $x, y \in R$.

$\left(\mathrm{H}_{4}\right)$ The delay kernels $k_{i j}:[0,+\infty) \rightarrow R$ are continuous, integrable, and satisfy

$$
\int_{0}^{+\infty}\left|k_{i j}(s)\right| \mathrm{d} s \leq \beta_{i j}, \quad i, j=1,2, \ldots, n
$$

$\left(\mathrm{H}_{5}\right)$ There exists a constant $\alpha_{0}>0$ such that

$$
\int_{0}^{+\infty}\left|k_{i j}(s)\right| e^{\alpha_{0} s} \mathrm{~d} s \leq+\infty, \quad i, j=1,2, \ldots, n
$$

The organization of this paper is as follows. In Section 2, we introduce some notations and definitions, and state some preliminary results needed in later sections. We then study, in Section 3, the existence of periodic solutions of system (1.1) by using the continuation theorem of coincidence degree theorem proposed by Gains and Mawhin [13]. In Section 4, by constructing Lyapunov function we will derive sufficient conditions for the global exponential stability of the periodic solution of system (1.1). At last, an example is employed to illustrate the feasible results of this paper.

\section{Preliminaries}

For convenience, we use $\bar{a}_{i j}, \bar{b}_{i j}, \bar{c}_{i j}, \bar{I}_{i}$ to denote the maximums of $\left|a_{i j}(t)\right|,\left|b_{i j}(t)\right|,\left|c_{i j}(t)\right|,\left|I_{i}(t)\right|$, respectively. We also use symbols $\tau=\max \left\{\tau_{i j}(t): t \in[0, \omega], i, j=1,2, \ldots, n\right\}, x=$ $\left(x_{1}, x_{2}, \ldots, x_{n}\right)^{T}$ to denote a column vector, in which the symbol $(T)$ denotes the transpose of a vector. $E_{n}$ denotes the identity matrix of size $n$. A matrix or vector $D \geq 0$ means that all entries of $D$ are greater than or equal to zero (resp., $D>0$ ). For matrices or vectors $D$ and $E, D \geq E$ (resp., $D>E$ ) means that $D-E \geq 0$ (resp., $D-E>0$ ). 
The initial condition $\phi=\left(\phi_{1}, \ldots, \phi_{n}\right)^{T}$ of $(1.1)$ is of the form

$$
x_{i}(s)=\phi_{i}(s), \quad s \in(-\infty, 0], i=1, \ldots, n,
$$

where $\phi_{i}(s), i=1,2, \ldots, n$, are continuous $\omega$-periodic solutions.

Definition 2.1. Let $x^{*}(t)$ be an $\omega$-periodic solution of (1.1) with initial value $\phi^{*}=\left(\phi_{1}^{*}, \ldots, \phi_{n}^{*}\right)^{T} \in$ $C\left((-\infty, 0] ; R^{n}\right)$. If there exist constants $\alpha>0$ and $P>1$ such that for every solution $x(t)$ of (1.1) with initial value $\phi \in C\left((-\infty, 0] ; R^{n}\right)$,

$$
\left|x_{i}(t)-x_{i}^{*}(t)\right| \leq P\left\|\phi-\phi^{*}\right\| e^{-\alpha t}, \quad \forall t>0, i=1,2, \ldots, n,
$$

where $\left\|\phi-\phi^{*}\right\|=\sup _{s \leq 0} \max _{1 \leq i \leq n}\left|\phi_{i}(s)-\phi_{i}^{*}(s)\right|$, then $x^{*}(t)$ is said to be globally exponentially stable.

Definition 2.2 (see $[15,16])$. A real $n \times n$ matrix $F=\left(f_{i j}\right)_{n \times n}$ is said to be a nonsingular $M$-matrix if $f_{i j} \leq 0, i, j=1,2, \ldots, n, i \neq j$, and $F^{-1} \geq 0$, where $F^{-1}$ denotes the inverse of $F$.

Lemma 2.3 (see $[15,16])$. Let $F=\left(f_{i j}\right)_{n \times n}$ with $f_{i j} \leq 0, i, j=1,2, \ldots, n, i \neq j$. Then the following statements are equivalent:

(1) $F$ is a nonsingular M-matrix,

(2) there exists a vector $\eta^{T}=\left(\eta_{1}, \eta_{2}, \ldots, \eta_{n}\right)>0$ such that $\eta F>0$,

(3) there exists a vector $\xi=\left(\xi_{1}, \xi_{2}, \ldots, \xi_{n}\right)^{T}>0$ such that $F \xi>0$.

Lemma 2.4 (see [16]). Let $A \geq 0$ be an $n \times n$ matrix and $\rho(A)<1$, then there exists a vector $\xi=\left(\xi_{1}, \xi_{2}, \ldots, \xi_{n}\right)^{T}>0$ such that $\left(E_{n}-A\right) \xi>0$, where $\rho(A)$ denotes the spectral radius of $A$. follows.

To end this section, we introduce Mawhin's continuation theorem [13, page 40] as

Consider an abstract equation in a Banach space $X$,

$$
L x=\lambda N x, \quad \lambda \in(0,1),
$$

where $L: \operatorname{Dom} L \cap X \rightarrow X$ is a Fredholm operator with index-zero, and $l$ is a parameter. Let $P$ and $Q$ denote two projectors, $P: \operatorname{Dom} L \cap X \rightarrow \operatorname{Ker} L$ and $Q: X \rightarrow X / \operatorname{Im} L$ such that $\operatorname{Im} P=$ $\operatorname{Ker} L, \operatorname{Ker} Q=\operatorname{Im} L$.

Lemma 2.5. Let $X$ be a Banach space. Suppose that $L:$ Dom $L \cap X \rightarrow X$ is a Fredholm operator with index-zero, let $\Omega \subset X$ be an open bounded set, and let $N: X \rightarrow X$ be a continuous operator which is $L$-compact on $\bar{\Omega}$. Moreover, assume that the following conditions are satisfied:

(a) for each $\lambda \in(0,1), x \in \partial \Omega \cap \operatorname{Dom} L, L x \neq \lambda N x$,

(b) for each $x \in \partial \Omega \cap \operatorname{Ker} L, Q N x \neq 0$,

(c) $\operatorname{deg}(Q N, \Omega \cap \operatorname{Ker} L, 0) \neq 0$.

Then, $L x=N x$ has at least one solution in $\bar{\Omega} \cap \operatorname{Dom} L$. 


\section{Existence of periodic solutions}

Theorem 3.1. Let $\left(H_{1}\right)-\left(H_{4}\right)$ hold. Assume that the following condition is satisfied:

$\left(\mathrm{H}_{6}\right)$ there exists a vector $\eta^{T}=\left(\eta_{1}, \ldots, \eta_{n}\right)>0$ such that

$$
\bar{\eta}^{T}=\left(\bar{\eta}_{1}, \ldots, \bar{\eta}_{n}\right)=\eta^{T}\left(E_{n}-\Gamma\right)>0,
$$

where $\Gamma=\left(\gamma_{i j}\right)_{n \times n}, \gamma_{i j}=\underline{a}_{i}^{-1}\left(\bar{a}_{i j} L_{j}^{f}+\bar{b}_{i j} L_{j}^{f}+\bar{c}_{i j} \beta_{i j} L_{j}^{g}\right), i, j=1,2, \ldots, n$.

Then, (1.1) has at least one w-periodic solution.

Proof. For convenience, we introduce the following notations:

$$
\begin{aligned}
G_{i}(t)=-a_{i}\left(t, x_{i}(t)\right)+\sum_{j=1}^{n}[ & a_{i j}(t) f_{j}\left(x_{j}(t)\right)+b_{i j}(t) f_{j}\left(x_{j}\left(t-\tau_{i j}(t)\right)\right) \\
& \left.+c_{i j}(t) \int_{-\infty}^{t} k_{i j}(t-s) g_{j}\left(x_{j}(s)\right) \mathrm{d} s\right]+I_{i}(t), \quad i=1,2, \ldots, n .
\end{aligned}
$$

In order to use Lemma 2.5, we take $X=\left\{x(t) \in C\left(R, R^{n}\right): x(t+\omega)=x(t), t \in R\right\}$; then $X$ is a Banach space with the norm

$$
\|x\|=\max \left\{\left|x_{1}\right|_{0^{\prime}}\left|x_{2}\right|_{0^{\prime}}, \ldots,\left|x_{n}\right|_{0}\right\}, \quad\left|x_{i}\right|_{0}=\sup _{t \in[0, \omega]}\left|x_{i}(t)\right|, \quad i=1,2, \ldots, n .
$$

Set

$$
\begin{aligned}
& L x=x^{\prime}, \quad P x=Q x=\frac{1}{\omega} \int_{0}^{\omega} x(t) \mathrm{d} t, \quad x \in X, \\
& N x=\left(G_{1}(t), G_{2}(t), \ldots, G_{n}(t)\right)^{T}, \quad x \in X .
\end{aligned}
$$

Obviously, $\operatorname{Ker} L=\left\{x \mid x \in X, x=h, h \in R^{n}\right\}, \operatorname{Im} L=\left\{x \mid x \in X, \int_{0}^{\omega} x(s) \mathrm{d} s=0\right\}$, and $\operatorname{dim} \operatorname{Ker} L=n=\operatorname{codim} \operatorname{Im} L$. So, $\operatorname{Im} L$ is closed in $X$. It is easy to show that $P$ and $Q$ are continuous projectors satisfying $\operatorname{Im} P=\operatorname{Ker} L, \operatorname{Im} L=\operatorname{Ker} Q=\operatorname{Im}(I-Q)$. Hence, $L$ is a Fredholm mapping of index-zero. Furthermore, through an easy computation, we find that the generalized inverse $K_{P}^{-1}: \operatorname{Im} L \rightarrow \operatorname{Ker} P \cap \operatorname{dom} L$ of $L$ has the form

$$
K_{P}^{-1}(x)=\int_{0}^{t} x(s) \mathrm{d} s-\frac{1}{\omega} \int_{0}^{\omega} \int_{0}^{t} x(s) \mathrm{d} s \mathrm{~d} t .
$$

Thus,

$$
\begin{aligned}
& Q N x=\left(\frac{1}{\omega} \int_{0}^{\omega} G_{1}(t) \mathrm{d} t, \ldots, \frac{1}{\omega} \int_{0}^{\omega} G_{n}(t) \mathrm{d} t\right)^{T}, \quad x \in X, \\
& K_{p}^{-1}(I-Q) N x=\left(\begin{array}{c}
\int_{0}^{t} G_{1}(s) \mathrm{d} s \\
\vdots \\
\int_{0}^{t} G_{j}(s) \mathrm{d} s \\
\vdots \\
\int_{0}^{t} G_{n}(s) \mathrm{d} s
\end{array}\right)-\left(\begin{array}{c}
\frac{1}{\omega} \int_{0}^{\omega} \int_{0}^{t} G_{1}(s) \mathrm{d} s \mathrm{~d} t \\
\vdots \\
\frac{1}{\omega} \int_{0}^{\omega} \int_{0}^{t} G_{j}(s) \mathrm{d} s \mathrm{~d} t \\
\vdots \\
\frac{1}{\omega} \int_{0}^{\omega} \int_{0}^{t} G_{n}(s) \mathrm{d} s \mathrm{~d} t
\end{array}\right)-\left(\begin{array}{c}
\left(\frac{t}{\omega}-\frac{1}{2}\right) \int_{0}^{\omega} G_{1}(s) \mathrm{d} s \\
\vdots \\
\left(\frac{t}{\omega}-\frac{1}{2}\right) \int_{0}^{\omega} G_{j}(s) \mathrm{d} s \\
\vdots \\
\left(\frac{t}{\omega}-\frac{1}{2}\right) \int_{0}^{\omega} G_{n}(s) \mathrm{d} s
\end{array}\right) .
\end{aligned}
$$


Clearly, $Q N$ and $K_{P}^{-1}(I-Q) N$ are continuous. Using the Arzela-Ascoli theorem, it is not difficult to show that $Q N(\bar{\Omega}), K_{P}^{-1}(I-Q) N(\bar{\Omega})$ are relatively compact for any open bounded set $\Omega \subset X$. Therefore, $N$ is $L$-compact on $\bar{\Omega}$ for any open bounded set $\Omega \subset X$.

Now, we reach the position to search for an appropriate open bounded subset $\Omega$ for the application of Lemma 2.5. Corresponding to the operator equation (2.3), we have

$$
\begin{array}{r}
x_{i}^{\prime}(t)=\lambda\left\{-a_{i}\left(t, x_{i}(t)\right)+\sum_{j=1}^{n}\left[a_{i j}(t) f_{j}\left(x_{j}(t)\right)+b_{i j}(t) f_{j}\left(x_{j}\left(t-\tau_{i j}(t)\right)\right)\right.\right. \\
\left.\left.+c_{i j}(t) \int_{-\infty}^{t} k_{i j}(t-s) g_{j}\left(x_{j}(s)\right) \mathrm{d} s\right]+I_{i}(t)\right\}, \\
i=1,2, \ldots, n, \lambda \in(0,1) .
\end{array}
$$

Let $x(t)=\left(x_{1}(t), x_{2}(t), \ldots, x_{n}(t)\right)^{T} \in X$ be a solution of system (3.7) for some $\lambda \in(0,1)$. Then, for any $i=1,2, \ldots, n, x_{i}$ are all continuously differentiable. Thus, there exist $t_{i} \in[0, \omega], i=$ $1,2, \ldots, n$, such that $\left|x_{i}\left(t_{i}\right)\right|=\max _{t \in[0, \omega]}\left|x_{i}(t)\right|$. Hence, $x_{i}^{\prime}\left(t_{i}\right)=0, i=1,2, \ldots, n$. From (3.7), we have

$$
\begin{aligned}
a_{i}\left(t_{i}, x_{i}\left(t_{i}\right)\right)=\sum_{j=1}^{n}[ & a_{i j}\left(t_{i}\right) f_{j}\left(x_{j}\left(t_{i}\right)\right)+b_{i j}\left(t_{i}\right) f_{j}\left(x_{j}\left(t_{i}-\tau_{i j}\left(t_{i}\right)\right)\right) \\
& \left.\quad+c_{i j}\left(t_{i}\right) \int_{-\infty}^{t_{i}} k_{i j}\left(t_{i}-s\right) g_{j}\left(x_{j}(s)\right) \mathrm{d} s\right]+I_{i}\left(t_{i}\right), \quad i=1,2, \ldots, n .
\end{aligned}
$$

In view of $\left(\mathrm{H}_{1}\right)-\left(\mathrm{H}_{4}\right)$, we have

$$
\begin{aligned}
\underline{a}_{i}\left|x_{i}\left(t_{i}\right)\right| \leq\left|a_{i}\left(t_{i}, x_{i}\left(t_{i}\right)\right)\right| \\
\leq \sum_{j=1}^{n}\left[\left|a_{i j}\left(t_{i}\right)\right|\left(\left|f_{j}\left(x_{j}\left(t_{i}\right)\right)-f_{j}(0)\right|+\left|f_{j}(0)\right|\right)\right. \\
\quad+\left|b_{i j}\left(t_{i}\right)\right|\left(\left|f_{j}\left(x_{j}\left(t_{i}-\tau_{i j}\left(t_{i}\right)\right)\right)-f_{j}(0)\right|+\left|f_{j}(0)\right|\right) \\
\left.\quad \quad+\left|c_{i j}\left(t_{i}\right)\right| \int_{-\infty}^{t_{i}}\left|k_{i j}\left(t_{i}-s\right)\right|\left(\left|g_{j}\left(x_{j}(s)\right)-g_{j}(0)\right|+\left|g_{j}(0)\right|\right) \mathrm{d} s\right]+\left|I_{i}\left(t_{i}\right)\right| \\
\leq \sum_{j=1}^{n}\left[\bar{a}_{i j} L_{j}^{f}\left|x_{j}\left(t_{i}\right)\right|+\bar{b}_{i j} L_{j}^{f}\left|x_{j}\left(t_{i}-\tau_{i j}\left(t_{i}\right)\right)\right|\right. \\
\left.\quad+\bar{c}_{i j} \int_{-\infty}^{t_{i}}\left|k_{i j}\left(t_{i}-s\right)\right|\left(L_{j}^{g}\left|x_{j}(s)\right|+\left|g_{j}(0)\right|\right) \mathrm{d} s+\left(\bar{a}_{i j}+\bar{b}_{i j}\right)\left|f_{j}(0)\right|\right]+\bar{I}_{i} \\
\leq \sum_{j=1}^{n}\left(\bar{a}_{i j} L_{j}^{f}+\bar{b}_{i j} L_{j}^{f}+\bar{c}_{i j} \beta_{i j} L_{j}^{g}\right)\left|x_{j}\left(t_{j}\right)\right| \\
\quad+\sum_{j=1}^{n}\left(\bar{a}_{i j}\left|f_{j}(0)\right|+\bar{b}_{i j}\left|f_{j}(0)\right|+\bar{c}_{i j} \beta_{i j}\left|g_{j}(0)\right|\right)+\bar{I}_{i}, \quad i=1,2, \ldots, n .
\end{aligned}
$$


Set $H_{i}=\sum_{j=1}^{n} \underline{a}_{i}^{-1}\left(\bar{a}_{i j}\left|f_{j}(0)\right|+\bar{b}_{i j}\left|f_{j}(0)\right|+\bar{c}_{i j} \beta_{i j}\left|g_{j}(0)\right|\right)+\underline{a}_{i}^{-1} \bar{I}_{i}$. Clearly, (3.9) implies that

$$
\left|x_{i}\left(t_{i}\right)\right| \leq \sum_{j=1}^{n} \gamma_{i j}\left|x_{j}\left(t_{j}\right)\right|+H_{i}, \quad i=1,2, \ldots, n .
$$

Thus,

$$
\left(E_{n}-\Gamma\right)\left(\left|x_{1}\left(t_{1}\right)\right|,\left|x_{2}\left(t_{2}\right)\right|, \ldots,\left|x_{n}\left(t_{n}\right)\right|\right)^{T} \leq\left(H_{1}, H_{2}, \ldots, H_{n}\right)^{T}:=H ;
$$

together with $\left(\mathrm{H}_{6}\right)$, we have

$$
\begin{aligned}
\min & \left\{\bar{\eta}_{1}, \bar{\eta}_{2}, \ldots, \bar{\eta}_{n}\right\}\left(\left|x_{1}\left(t_{1}\right)\right|+\left|x_{2}\left(t_{2}\right)\right|+\cdots+\left|x_{n}\left(t_{n}\right)\right|\right) \\
& \leq \bar{\eta}_{1}\left|x_{1}\left(t_{1}\right)\right|+\bar{\eta}_{2}\left|x_{2}\left(t_{2}\right)\right|+\cdots+\bar{\eta}_{n}\left|x_{n}\left(t_{n}\right)\right| \\
& =\eta^{T}\left(E_{n}-\Gamma\right)\left(\left|x_{1}\left(t_{1}\right)\right|,\left|x_{2}\left(t_{2}\right)\right|, \ldots,\left|x_{n}\left(t_{n}\right)\right|\right)^{T} \leq \eta^{T} H .
\end{aligned}
$$

Therefore,

$$
\left|x_{i}\right|_{0}=\left|x_{i}\left(t_{i}\right)\right| \leq \frac{\eta^{T} H}{\min \left\{\bar{\eta}_{1}, \bar{\eta}_{2}, \ldots, \bar{\eta}_{n}\right\}}: v, \quad i=1,2, \ldots, n .
$$

Again from $\left(\mathrm{H}_{6}\right)$, it follows from Lemma 2.3 that $E_{n}-\Gamma$ is a nonsingular $M$-matrix, and there exists a vector $\xi=\left(\xi_{1}, \xi_{2}, \ldots, \xi_{n}\right)^{T}>0$ such that $\left(E_{n}-\Gamma\right) \xi>0$, which implies that we can choose a constant $d>1$ such that $\bar{\xi}=\left(\bar{\xi}_{1}, \bar{\xi}_{2}, \ldots, \bar{\xi}_{n}\right)^{T}=d \xi$ and $\bar{\xi}_{i}=d \xi_{i}>v, i=1,2, \ldots, n$, and

$$
\left(E_{n}-\Gamma\right) \bar{\xi}=d\left(E_{n}-\Gamma\right) \xi>H \text {. }
$$

We take

$$
\Omega=\{x(t) \in X:-\bar{\xi}<x(t)<\bar{\xi}, \forall t \in R\},
$$

which satisfies Condition (a) of Lemma 2.5. If $x(t) \in \partial \Omega \cap \operatorname{ker} L$, then $x(t)$ is a constant vector in $R^{n}$, and there exists some $i \in\{1,2, \ldots, n\}$ such that $\left|x_{i}\right|=\bar{\xi}_{i}$. We claim that

$$
\left|(Q N x)_{i}\right|>0, \quad x \in \partial \Omega \cap \operatorname{ker} L .
$$

By way of contradiction, suppose that $\left|(Q N x)_{i}\right|=0$; then there exists some $t^{*} \in[0, \omega]$ such that

$$
-a_{i}\left(t^{*}, x_{i}\right)+\sum_{j=1}^{n}\left[a_{i j}\left(t^{*}\right) f_{j}\left(x_{j}\right)+b_{i j}\left(t^{*}\right) f_{j}\left(x_{j}\right)+c_{i j}\left(t^{*}\right) \int_{-\infty}^{t^{*}} k_{i j}\left(t^{*}-s\right) g_{j}\left(x_{j}(s)\right) \mathrm{d} s\right]+I_{i}\left(t^{*}\right)=0,
$$

which implies that

$$
\begin{aligned}
\underline{a}_{i} \bar{\xi}_{i} & =\underline{a}_{i}\left|x_{i}\right| \leq\left|a_{i}\left(t^{*}, x_{i}\right)\right| \\
& =\left|\sum_{j=1}^{n}\left[a_{i j}\left(t^{*}\right) f_{j}\left(x_{j}\right)+b_{i j}\left(t^{*}\right) f_{j}\left(x_{j}\right)+c_{i j}\left(t^{*}\right) \int_{-\infty}^{t^{*}} k_{i j}\left(t^{*}-s\right) g_{j}\left(x_{j}(s)\right) \mathrm{d} s\right]+I_{i}\left(t^{*}\right)\right| \\
& \leq \sum_{j=1}^{n}\left(\bar{a}_{i j} L_{j}^{f}+\bar{b}_{i j} L_{j}^{f}+\bar{c}_{i j} \beta_{i j} L_{j}^{g}\right)\left|x_{j}\right|+\sum_{j=1}^{n}\left(\bar{a}_{i j}\left|f_{j}(0)\right|+\bar{b}_{i j}\left|f_{j}(0)\right|+\bar{c}_{i j} \beta_{i j}\left|g_{j}(0)\right|\right)+\bar{I}_{i} \\
& \leq \sum_{j=1}^{n}\left(\bar{a}_{i j} L_{j}^{f}+\bar{b}_{i j} L_{j}^{f}+\bar{c}_{i j} \beta_{i j} L_{j}^{g}\right) \bar{\xi}_{j}+\sum_{j=1}^{n}\left(\bar{a}_{i j}\left|f_{j}(0)\right|+\bar{b}_{i j}\left|f_{j}(0)\right|+\bar{c}_{i j} \beta_{i j}\left|g_{j}(0)\right|\right)+\bar{I}_{i} ;
\end{aligned}
$$


hence

$$
\bar{\xi}_{i} \leq \sum_{j=1}^{n} \gamma_{i j} \bar{\xi}_{j}+H_{i}
$$

This implies that $\left(\left(E_{n}-\Gamma\right) \bar{\xi}\right)_{i} \leq H_{i}$, which contradicts (3.14). Therefore, (3.16) holds, and hence, Condition (b) of Lemma 2.5 is satisfied.

Furthermore, we define a continuous function $W: \Omega \cap \operatorname{Ker} L \times[0,1] \rightarrow X$ by

$$
W(x, \mu)=\mu \operatorname{diag}\left(-\bar{a}_{1},-\bar{a}_{2}, \ldots,-\bar{a}_{n}\right) x+(1-\mu) Q N x,
$$

for all $x \in \Omega \cap \operatorname{Ker} L=\Omega \cap R^{n}$ and $\mu \in[0,1]$. It follows that

$$
(W(x, \mu))_{i}=-\mu \bar{a}_{i} x_{i}+(1-\mu) \frac{1}{\omega} \int_{0}^{\omega} G_{i}(t) \mathrm{d} t .
$$

If $x(t) \in \partial \Omega \cap \operatorname{Ker} L$, then $x(t)$ is a constant vector in $R^{n}$, and there exists some $i \in$ $\{1,2, \ldots, n\}$ such that $\left|x_{i}\right|=\bar{\xi}_{i}$. We claim that

$$
\left|(W(x, \mu))_{i}\right|>0, \quad x \in \partial \Omega \cap \operatorname{ker} L, \mu \in[0,1] .
$$

By way of contradiction, suppose that $\left|(W(x, \mu))_{i}\right|=0$; then there exists some $\tilde{t} \in[0, \omega]$ such that

$$
\begin{aligned}
-\mu \bar{a}_{i} x_{i}+(1-\mu)\left\{-a_{i}\left(\tilde{t}, x_{i}\right)+\sum_{j=1}^{n}\right. & {\left[a_{i j}(\tilde{t}) f_{j}\left(x_{j}\right)+b_{i j}(\tilde{t}) f_{j}\left(x_{j}\right)\right.} \\
& \left.\left.+c_{i j}(\tilde{t}) \int_{-\infty}^{\tilde{t}} k_{i j}(\tilde{t}-s) g_{j}\left(x_{j}(s)\right) \mathrm{d} s\right]+I_{i}(\tilde{t})\right\}=0,
\end{aligned}
$$

that is,

$$
\begin{aligned}
& \mu\left(a_{i}\left(\tilde{t}, x_{i}\right)-\bar{a}_{i} x_{i}\right)-a_{i}\left(\tilde{t}, x_{i}\right) \\
& \quad=-(1-\mu)\left\{\sum_{j=1}^{n}\left[a_{i j}(\tilde{t}) f_{j}\left(x_{j}\right)+b_{i j}(\tilde{t}) f_{j}\left(x_{j}\right)+c_{i j}(\tilde{t}) \int_{-\infty}^{\tilde{t}} k_{i j}(\tilde{t}-s) g_{j}\left(x_{j}(s)\right) \mathrm{d} s\right]+I_{i}(\tilde{t})\right\} .
\end{aligned}
$$

Now, we will consider the following two cases.

Case 1. If $x_{i}>0$, from $\left(\mathrm{H}_{2}\right)$, we have

$$
a_{i}\left(\tilde{t}, x_{i}\right)>0, \quad a_{i}\left(\tilde{t}, x_{i}\right)-\bar{a}_{i} x_{i} \leq 0 .
$$

Then, from (3.24), we have

$$
a_{i}\left(\tilde{t}, x_{i}\right) \leq(1-\mu)\left\{\sum_{j=1}^{n}\left[a_{i j}(\tilde{t}) f_{j}\left(x_{j}\right)+b_{i j}(\tilde{t}) f_{j}\left(x_{j}\right)+c_{i j}(\tilde{t}) \int_{-\infty}^{\tilde{t}} k_{i j}(\tilde{t}-s) g_{j}\left(x_{j}(s)\right) \mathrm{d} s\right]+I_{i}(\tilde{t})\right\},
$$


which implies that

$$
\begin{aligned}
\underline{a}_{i} \bar{\xi}_{i} & =\underline{a}_{i}\left|x_{i}\right| \leq\left|a_{i}\left(\tilde{t}, x_{i}\right)\right| \\
& \leq\left|\sum_{j=1}^{n}\left[a_{i j}(\tilde{t}) f_{j}\left(x_{j}\right)+b_{i j}(\tilde{t}) f_{j}\left(x_{j}\right)+c_{i j}(\tilde{t}) \int_{-\infty}^{\tilde{t}} k_{i j}(\tilde{t}-s) g_{j}\left(x_{j}(s)\right) \mathrm{d} s\right]+I_{i}(\tilde{t})\right| \\
& \leq \sum_{j=1}^{n}\left(\bar{a}_{i j} L_{j}^{f}+\bar{b}_{i j} L_{j}^{f}+\bar{c}_{i j} \beta_{i j} L_{j}^{g}\right)\left|x_{j}\right|+\sum_{j=1}^{n}\left(\bar{a}_{i j}\left|f_{j}(0)\right|+\bar{b}_{i j}\left|f_{j}(0)\right|+\bar{c}_{i j} \beta_{i j}\left|g_{j}(0)\right|\right)+\bar{I}_{i} \\
& \leq \sum_{j=1}^{n}\left(\bar{a}_{i j} L_{j}^{f}+\bar{b}_{i j} L_{j}^{f}+\bar{c}_{i j} \beta_{i j} L_{j}^{g}\right) \bar{\xi}_{j}+\sum_{j=1}^{n}\left(\bar{a}_{i j}\left|f_{j}(0)\right|+\bar{b}_{i j}\left|f_{j}(0)\right|+\bar{c}_{i j} \beta_{i j}\left|g_{j}(0)\right|\right)+\bar{I}_{i} .
\end{aligned}
$$

Hence,

$$
\bar{\xi}_{i} \leq \sum_{j=1}^{n} \gamma_{i j} \bar{\xi}_{j}+H_{i}
$$

this implies that $\left(\left(E_{n}-\Gamma\right) \bar{\xi}\right)_{i} \leq H_{i}$, which contradicts (3.14). Therefore, (3.22) holds.

Case 2. If $x_{i}<0$, from $\left(\mathrm{H}_{2}\right)$, we have

$$
a_{i}\left(\tilde{t}, x_{i}\right)<0, \quad a_{i}\left(\tilde{t}, x_{i}\right)-\bar{a}_{i} x_{i} \geq 0 .
$$

Then, from (3.24), we have

$$
a_{i}\left(\tilde{t}, x_{i}\right) \geq(1-\mu)\left\{\sum_{j=1}^{n}\left[a_{i j}(\tilde{t}) f_{j}\left(x_{j}\right)+b_{i j}(\tilde{t}) f_{j}\left(x_{j}\right)+c_{i j}(\tilde{t}) \int_{-\infty}^{\tilde{t}} k_{i j}(\tilde{t}-s) g_{j}\left(x_{j}\right) \mathrm{d} s\right]+I_{i}(\tilde{t})\right\} .
$$

The later proof is similar to that of Case 1. We can also show that (3.22) holds. It follows that $W(x, \mu) \neq 0$ for $x \in \partial \Omega \cap \operatorname{Ker} L, \mu \in[0,1]$. Hence, by homotopy invariance theorem and $\bar{a}_{i}>0, i=1,2, \ldots, n$, we obtain $\operatorname{deg}\{Q N, \Omega \cap \operatorname{ker} L, 0\}=\operatorname{deg}\left\{\left(-\bar{a}_{1} x_{1},-\bar{a}_{2} x_{2}, \ldots,-\bar{a}_{n} x_{n}\right)^{T}, \Omega \cap\right.$ ker $L, 0\} \neq 0$ for $x \in \partial \Omega \cap \operatorname{Ker} L$. Till now, we have proved that $\Omega$ satisfies all conditions of Lemma 2.5. Therefore, (1.1) has a periodic solution $x^{*}(t)$. This completes the proof.

Corollary 3.2. Let $\left(H_{1}\right)-\left(H_{4}\right)$ hold. Assume that $E_{n}-\Gamma$ is a nonsingular M-matrix, then (1.1) has at least one $\omega$-periodic solution, where $\Gamma$ is defined as above.

Corollary 3.3. Let $\left(H_{1}\right)-\left(H_{4}\right)$ hold. Assume that $\rho(\Gamma)<1$, then $(1.1)$ has at least one w-periodic solution, where $\Gamma$ is defined as above.

Proof. Obviously, $\Gamma \geq 0$. By $\rho(\Gamma)<1$ and from Lemma 2.4, there exists a vector $\xi=$ $\left(\xi_{1}, \xi_{2}, \ldots, \xi_{n}\right)^{T}>0$ such that $\left(E_{n}-A\right) \xi>0$. The remaining part of the proof is the same as that of Theorem 3.1. 


\section{Global exponential stability of periodic solutions}

In this section, we will construct some suitable Lyapunov functionals to derive sufficient conditions which ensure that (1.1) has a unique $\omega$-periodic solution, and all solutions of (1.1) exponentially converge to its unique $\omega$-periodic solution.

Theorem 4.1. Assume that $\left(H_{1}\right)-\left(H_{6}\right)$ hold and

$\left(\mathrm{H}_{7}\right)\left|a_{i}(t, u)-a_{i}(t, v)\right| \geq \underline{a}_{i}|u-v|$, for all $t, u, v \in R, i=1,2, \ldots, n$, where $\underline{a}_{i}, i=1,2, \ldots, n$, are the positive constants of Hypothesis $\left(H_{2}\right)$. Then, (1.1) has exactly one $\omega$-periodic solution, which is globally exponentially stable.

Proof. By Theorem 3.1, there exists an $\omega$-periodic solution $x^{*}(t)=\left(x_{1}^{*}(t), x_{2}^{*}(t), \ldots, x_{n}^{*}(t)\right)^{T}$ of (1.1). Suppose that $x(t)$ is an arbitrary solution of (1.1). Set $z(t)=\left(z_{1}(t), z_{2}(t), \ldots, z_{n}(t)\right)^{T}=$ $x(t)-x^{*}(t)$. Then,

$$
\begin{aligned}
z_{i}^{\prime}(t)=-d_{i}\left(t, z_{i}(t)\right)+\sum_{j=1}^{n} & {\left[a_{i j}(t) w_{j}\left(z_{j}(t)\right)+b_{i j}(t) w_{j}\left(z_{j}\left(t-\tau_{i j}(t)\right)\right)\right.} \\
& \left.+c_{i j}(t) \int_{-\infty}^{t} k_{i j}(t-s) u_{j}\left(z_{j}(s)\right) \mathrm{d} s\right], \quad i=1,2, \ldots, n
\end{aligned}
$$

where

$$
\begin{aligned}
& d_{i}\left(t, z_{i}(t)\right)=a_{i}\left(t, z_{i}(t)+x_{i}^{*}(t)\right)-a_{i}\left(t, x_{i}^{*}(t)\right), \\
& w_{j}\left(z_{j}(t)\right)=f_{j}\left(z_{j}(t)+x_{j}^{*}(t)\right)-f_{j}\left(x_{j}^{*}(t)\right), \\
& w_{j}\left(z_{j}\left(t-\tau_{i j}(t)\right)\right)=f_{j}\left(z_{j}\left(t-\tau_{i j}(t)\right)+x_{j}^{*}\left(t-\tau_{i j}(t)\right)\right)-f_{j}\left(x_{j}^{*}\left(t-\tau_{i j}(t)\right)\right), \\
& u_{j}\left(z_{j}(t)\right)=g_{j}\left(z_{j}(t)+x_{j}^{*}(t)\right)-g_{j}\left(x_{j}^{*}(t)\right) .
\end{aligned}
$$

From $\left(\mathrm{H}_{6}\right)$, it follows from Lemma 2.3 that $E_{n}-\Gamma$ is a nonsingular $M$-matrix, and there exists a vector $\xi=\left(\xi_{1}, \xi_{2}, \ldots, \xi_{n}\right)^{T}>0$ such that $\left(E_{n}-\Gamma\right) \xi>0$. Then,

$$
-\underline{a}_{i} \xi_{i}+\sum_{j=1}^{n} \xi_{j}\left(\bar{a}_{i j} L_{j}^{f}+\bar{b}_{i j} L_{j}^{f}+\bar{c}_{i j} \beta_{i j} L_{j}^{g}\right)<0, \quad i=1,2, \ldots, n .
$$

Set

$$
h_{i}(\lambda)=\left(\lambda-\underline{a}_{i}\right) \xi_{i}+\sum_{j=1}^{n} \xi_{j}\left(\bar{a}_{i j} L_{j}^{f}+e^{\lambda \tau} \bar{b}_{i j} L_{j}^{f}+\bar{c}_{i j} L_{j}^{g} \int_{0}^{+\infty}\left|k_{i j}(s)\right| e^{\lambda s} \mathrm{~d} s\right), \quad i=1,2, \ldots, n .
$$

Clearly, $h_{i}(\lambda), i=1,2, \ldots, n$, are continuous functions on $\left[0, \alpha_{0}\right]$, where $\alpha_{0}$ is the positive constant of Hypothesis $\left(\mathrm{H}_{5}\right)$. Since

$$
\begin{aligned}
h_{i}(0) & =-\underline{a}_{i} \xi_{i}+\sum_{j=1}^{n} \xi_{j}\left(\bar{a}_{i j} L_{j}^{f}+\bar{b}_{i j} L_{j}^{f}+\bar{c}_{i j} L_{j}^{g} \int_{0}^{+\infty}\left|k_{i j}(s)\right| \mathrm{d} s\right) \\
& \leq-\underline{a}_{i} \xi_{i}+\sum_{j=1}^{n} \xi_{j}\left(\bar{a}_{i j} L_{j}^{f}+\bar{b}_{i j} L_{j}^{f}+\bar{c}_{i j} \beta_{i j} L_{j}^{g}\right)<0,
\end{aligned}
$$


we can choose a positive constant $\alpha \in\left(0, \alpha_{0}\right]$ such that

$$
h_{i}(\alpha)=\left(\alpha-\underline{a}_{i}\right) \xi_{i}+\sum_{j=1}^{n} \xi_{j}\left(\bar{a}_{i j} L_{j}^{f}+e^{\alpha \tau} \bar{b}_{i j} L_{j}^{f}+\bar{c}_{i j} L_{j}^{g} \int_{0}^{+\infty}\left|k_{i j}(s)\right| e^{\alpha s} \mathrm{~d} s\right)<0, \quad i=1,2, \ldots, n .
$$

Now, we choose a positive constant $d$ such that

$$
d \xi_{i} e^{-\alpha t}>1, \quad \text { for } t \in(-\infty, 0], i=1,2, \ldots, n .
$$

Define a Lyapunov function $V=\left(V_{1}, V_{2}, \ldots, V_{n}\right)^{T}$ by $V_{i}=e^{\alpha t}\left|z_{i}(t)\right|, i=1,2, \ldots, n$. In view of (4.1), we obtain

$$
\begin{aligned}
& \frac{\mathrm{d}^{+} V_{i}(t)}{\mathrm{d} t}=e^{\alpha t} \operatorname{sgn} z_{i}\left\{-d_{i}\left(t, z_{i}(t)\right)+\sum_{j=1}^{n}\left[a_{i j}(t) w_{j}\left(z_{j}(t)\right)+b_{i j}(t) w_{j}\left(z_{j}\left(t-\tau_{i j}(t)\right)\right)\right.\right. \\
& \left.\left.+c_{i j}(t) \int_{-\infty}^{t} k_{i j}(t-s) u_{j}\left(z_{j}(s)\right) \mathrm{d} s\right]\right\}+\alpha e^{\alpha t}\left|z_{i}(t)\right| \\
& \leq e^{\alpha t}\left\{\left(\alpha-\underline{a}_{i}\right)\left|z_{i}(t)\right|+\sum_{j=1}^{n}\left[\bar{a}_{i j} L_{j}^{f}\left|z_{j}(t)\right|+\bar{b}_{i j} L_{j}^{f}\left|z_{j}\left(t-\tau_{i j}(t)\right)\right|\right.\right. \\
& \left.\left.+\bar{c}_{i j} L_{j}^{g} \int_{-\infty}^{t}\left|k_{i j}(t-s)\right|\left|z_{j}(s)\right| \mathrm{d} s\right]\right\} \\
& =\left(\alpha-\underline{a}_{i}\right) e^{\alpha t}\left|z_{i}(t)\right|+\sum_{j=1}^{n}\left[\bar{a}_{i j} L_{j}^{f} e^{\alpha t}\left|z_{j}(t)\right|+e^{\alpha \tau_{i j}(t)} \bar{b}_{i j} L_{j}^{f} e^{\alpha\left(t-\tau_{i j}(t)\right)}\left|z_{j}\left(t-\tau_{i j}(t)\right)\right|\right. \\
& \left.+\bar{c}_{i j} L_{j}^{g} \int_{-\infty}^{t}\left|k_{i j}(t-s)\right| e^{\alpha(t-s)} e^{\alpha s}\left|z_{j}(s)\right| \mathrm{d} s\right] \\
& \leq\left(\alpha-\underline{a}_{i}\right) V_{i}(t)+\sum_{j=1}^{n}\left[\bar{a}_{i j} L_{j}^{f} V_{j}(t)+e^{\alpha \tau} \bar{b}_{i j} L_{j}^{f} V_{j}\left(t-\tau_{i j}(t)\right)\right. \\
& \left.+\bar{c}_{i j} L_{j}^{g} \int_{-\infty}^{t}\left|k_{i j}(t-s)\right| e^{\alpha(t-s)} V_{j}(s) \mathrm{d} s\right], \quad i=1,2, \ldots, n .
\end{aligned}
$$

From (4.7), we have

$$
d \xi_{i}\left\|\phi-\phi^{*}\right\| e^{-\alpha t}>\left\|\phi-\phi^{*}\right\| \geq\left|z_{i}(t)\right|, \quad \text { for } t \in(-\infty, 0], i=1,2, \ldots, n,
$$

which implies that

$$
\left|z_{i}(t)\right|<d \xi_{i}\left\|\phi-\phi^{*}\right\| e^{-\alpha t}, \quad \text { for } t \in(-\infty, 0], i=1,2, \ldots, n,
$$

where $\left\|\phi-\phi^{*}\right\|$ is defined as that in Definition 2.1.

We claim that

$$
V_{i}(t)=\left|z_{i}(t)\right| e^{\alpha t}<d \xi_{i}\left\|\phi-\phi^{*}\right\|, \quad i=1,2, \ldots, n, \forall t>0 .
$$


Xinsong Yang

Contrarily, there must exist $i_{0} \in\{1,2, \ldots, n\}$ and $\tilde{t}>0$ such that

$$
\begin{aligned}
V_{i_{0}}(\tilde{t}) & =d \xi_{i_{0}}\left\|\phi-\phi^{*}\right\|, \\
V_{\theta}(\tilde{t}) & \leq d \xi_{\theta}\left\|\phi-\phi^{*}\right\|, \quad \theta=1,2, \ldots, i_{0}-1, i_{0}+1, \ldots, n, \\
V_{i}(t) & <d \xi_{i}\left\|\phi-\phi^{*}\right\|, \quad \forall t \in(-\infty, \tilde{t}), i=1,2, \ldots, n .
\end{aligned}
$$

Together with (4.8) and (4.12), we obtain

$$
\begin{aligned}
0 & \leq \frac{\mathrm{d}^{+}\left(V_{i_{0}}(\tilde{t})-d \xi_{i_{0}}\left\|\phi-\phi^{*}\right\|\right)}{\mathrm{d} t}=\frac{\mathrm{d}^{+} V_{i_{0}}(\tilde{t})}{\mathrm{d} t} \\
& \leq\left(\alpha-\underline{a}_{i_{0}}\right) V_{i_{0}}(\tilde{t})+\sum_{j=1}^{n}\left[\bar{a}_{i_{0} j} L_{j}^{f} V_{j}(\tilde{t})+e^{\alpha \tau} \bar{b}_{i_{0} j} L_{j}^{f} V_{j}\left(\tilde{t}-\tau_{i_{0} j}(\tilde{t})\right)+\bar{c}_{i_{0} j} L_{j}^{g} \int_{-\infty}^{\tilde{t}}\left|k_{i_{0} j}(\tilde{t}-s)\right| e^{\alpha(\tilde{t}-s)} V_{j}(s) \mathrm{d} s\right] \\
& \leq\left(\alpha-\underline{a}_{i_{0}}\right) d \xi_{i_{0}}\left\|\phi-\phi^{*}\right\|+\sum_{j=1}^{n} d \xi_{j}\left\|\phi-\phi^{*}\right\|\left(\bar{a}_{i_{0} j} L_{j}^{f}+e^{\alpha \tau} \bar{b}_{i_{0} j} L_{j}^{f}+\bar{c}_{i_{0} j} L_{j}^{g} \int_{0}^{+\infty}\left|k_{i_{0} j}(s)\right| e^{\alpha s} \mathrm{~d} s\right) .
\end{aligned}
$$

Hence,

$$
0 \leq\left(\alpha-\underline{a}_{i_{0}}\right) \xi_{i_{0}}+\sum_{j=1}^{n} \xi_{j}\left(\bar{a}_{i_{0} j} L_{j}^{f}+e^{\alpha \tau} \bar{b}_{i_{0} j} L_{j}^{f}+\bar{c}_{i_{0} j} L_{j}^{g} \int_{0}^{+\infty}\left|k_{i_{0} j}(s)\right| e^{\alpha s} \mathrm{~d} s\right),
$$

which contradicts (4.6). Therefore, (4.11) holds. It follows that

$$
\left|z_{i}(t)\right|<d \xi_{i}\left\|\phi-\phi^{*}\right\| e^{-\alpha t}, \quad t>0, i=1,2, \ldots, n .
$$

Let $M=\max _{1 \leq i \leq n}\left\{d \xi_{i}+1\right\}$. Then, from (4.15), we get

$$
\left|x_{i}(t)-x_{i}^{*}(t)\right|=\left|z_{i}(t)\right| \leq M\left\|\phi-\phi^{*}\right\| e^{-\alpha t}, \quad \forall t>0, i=1,2, \ldots, n .
$$

In view of Definition 2.1, the $\omega$-periodic solution $x^{*}(t)$ of system (1.1) is globally exponentially stable. This completes the proof.

Corollary 4.2. Let $\left(H_{1}\right)-\left(H_{7}\right)$ hold. Assume that $E_{n}-\Gamma$ is a nonsingular M-matrix or $\rho(\Gamma)<1$. Then, system (1.1) has exactly one w-periodic solution, which is globally exponentially stable, where $\Gamma$ is defined as that in $\left(H_{6}\right)$.

Remark 4.3. As a special case, $a_{i}\left(t, x_{i}(t)\right)=a_{i}(t) x_{i}(t)$ and $a_{i}(t)>0$. Let $\underline{a}_{i}=\min \left\{\left|a_{i}(t)\right|: t \in\right.$ $[0, \omega]\}, \bar{a}_{i}=\max \left\{\left|a_{i}(t)\right|: t \in[0, \omega]\right\}$, then $L_{i}^{a}=\underline{a}_{i}, i=1,2, \ldots, n$. Obviously, $\left(\mathrm{H}_{2}\right)$ and $\left(\mathrm{H}_{7}\right)$ hold.

Remark 4.4. When $a_{i}\left(t, x_{i}(t)\right)=c_{i} x_{i}(t), c_{i}$ are constants, and $c_{i j} \equiv 0, i, j=1,2, \ldots, n$, one can easily know that [11, Theorems 1 and 2] are direct corollaries of Theorems 3.1 and 4.1 of this paper, respectively. Moreover, we need not the following assumption:

$\left(\mathrm{H}_{0}\right) f_{j}(0)=0, g_{j}(0)=0,0 \leq \tau_{i j}^{\prime}(t)<1, i, j=1,2, \ldots, n$. 
Very recently, Zhou and $\mathrm{Hu}$ [12] considered the global exponential periodicity and stability of the following cellular neural networks:

$$
\begin{aligned}
x_{i}^{\prime}(t)=-d_{i} x_{i}(t)+\sum_{j=1}^{n} & {\left[a_{i j} f_{j}\left(x_{j}(t)\right)+b_{i j} f_{j}\left(x_{j}\left(t-\tau_{i j}(t)\right)\right)\right.} \\
& \left.+c_{i j} \int_{-\infty}^{t} k_{i j}(t-s) g_{j}\left(x_{j}(s)\right) \mathrm{d} s\right]+I_{i}(t), \quad i=1,2, \ldots, n,
\end{aligned}
$$

where $d_{i}>0, a_{i j}, b_{i j}, c_{i j}, i, j=1,2, \ldots, n$, are constants, $I_{i}(t+\omega)=I_{i}(t), i, j=1,2, \ldots, n, \tau_{i j}^{\prime}(t)<$ 1 , and $0 \leq \tau_{i j}(t) \leq \tau$. The assumptions in [12] on the delay kernels $k_{i j}$ of (4.17) are as follows.

$\left(\mathrm{F}_{1}\right)$ The delay kernels $k_{i j}: R^{+} \rightarrow R^{+}$are real-valued nonnegative continuous functions and $\int_{0}^{+\infty} k_{i j}(s) \mathrm{d} s=1, i, j=1,2, \ldots, n$.

$\left(\mathrm{F}_{2}\right) \int_{0}^{+\infty} s k_{i j}(s) \mathrm{d} s \leq+\infty, i, j=1,2, \ldots, n$.

$\left(\mathrm{F}_{3}\right)$ There exists a positive number $\mu$ such that $\int_{0}^{+\infty} e^{\mu s} k_{i j}(s) \mathrm{d} s \leq+\infty, i, j=1,2, \ldots, n$.

Obviously, (4.17) is a special case of (1.1). Moreover, (3.1) of Theorem 3.1 and (3.15) of Theorem 3.2 in [12] are special cases of (4.3) in this paper, that is, $\xi=(1,1, \ldots, 1)^{T}, L_{j}^{f}=L_{j}^{g}$, and $\beta_{i j}=1$. So, [12, Theorems 3.1 and 3.2] are special cases of Theorem 4.1 in this paper. To summarize, the results of this paper are completely new and generalize the results of [4-12] and the references cited therein.

\section{Application}

In this section, we give an example to illustrate that our results are feasible. Consider the following simple DCNNs with time-varying delays:

$$
\begin{aligned}
x_{i}^{\prime}(t)=-a_{i}\left(t, x_{i}(t)\right)+\sum_{j=1}^{2} & {\left[a_{i j}(t) f_{j}\left(x_{j}(t)\right)+b_{i j}(t) f_{j}\left(x_{j}\left(t-\tau_{i j}(t)\right)\right)\right.} \\
& \left.+c_{i j}(t) \int_{-\infty}^{t} k_{i j}(t-s) g_{j}\left(x_{j}(s)\right) \mathrm{d} s\right]+I_{i}(t), \quad i=1,2,
\end{aligned}
$$

where $f_{j}(x)=g_{j}(x)=|x|, a_{i}(t, x)=2 x+\sin x, I_{i}(t)=\sin 40 \pi t, \tau_{i j}(t)=(\cos 20 \pi t)^{2}, k_{i j}(t)=$ $\sin t e^{-t}$, and $a_{i j}(t)=b_{i j}(t)=c_{i j}(t)=(1 / 12) \cos 40 \pi t, i, j=1,2$. Then, we have

$$
\omega=\frac{1}{20}, \quad L_{j}^{f}=L_{j}^{g}=1, \quad \underline{a}_{i}=1, \quad \bar{a}_{i}=3, \quad \beta_{i j}=1, \quad \bar{a}_{i j}=\bar{b}_{i j}=\bar{c}_{i j}=\frac{1}{12}, \quad i, j=1,2 .
$$

Hence,

$$
\Gamma=\left(\begin{array}{ll}
\frac{1}{4} & \frac{1}{4} \\
\frac{1}{4} & \frac{1}{4}
\end{array}\right) .
$$


We take $\eta^{T}=\left(\eta_{1}, \eta_{2}\right)=(1,1)$, then

$$
\bar{\eta}=\left(\begin{array}{ll}
1 & 1
\end{array}\right)\left(\begin{array}{cc}
\frac{3}{4} & -\frac{1}{4} \\
-\frac{1}{4} & \frac{3}{4}
\end{array}\right)=\left(\begin{array}{ll}
\frac{1}{2} & \frac{1}{2}
\end{array}\right)>\left(\begin{array}{ll}
0 & 0
\end{array}\right) .
$$

It is easy to check that all the conditions needed in Theorem 4.1 are satisfied. Therefore, (5.1) has a unique global exponential 1/20-periodic solution.

Remark 5.1. Because $a_{i}\left(t, x_{i}(t)\right)$ is not linear about $x_{i}(t)$, thus none of the results in $[4-12,14]$ can be applied to (5.1). This implies that the results of this paper are new.

\section{Acknowledgments}

The author would like to express his gratitude to some anonymous reviewers and the editor for their valuable suggestions and comments. This work was supported by the Scientific Research Fund of Yunnan Provincial Education Department (07Y10085) and the NSF of Honghe University (XSS06009) of China.

\section{References}

[1] L. O. Chua and T. Roska, "Cellular neural networks with nonlinear and delay-type template elements," in Proceedings of the IEEE International Workshop on Cellular Neural Networks and Their Applications (CNNA '90), pp. 12-25, Budapest, Hungary, December 1990.

[2] L. O. Chua and L. Yang, "Cellular neural networks: theory," IEEE Transactions on Circuits and Systems, vol. 35, no. 10, pp. 1257-1272, 1988.

[3] P. L. Venetianer and T. Roska, "Image compression by cellular neural networks," IEEE Transactions on Circuits and Systems I, vol. 45, no. 3, pp. 205-215, 1998.

[4] X. Liao, G. Chen, and E. N. Sanchez, "Delay-dependent exponential stability analysis of delayed neural networks: an LMI approach," Neural Networks, vol. 15, no. 7, pp. 855-866, 2002.

[5] Z. Zeng, J. Wang, and X. Liao, "Global exponential stability of a general class of recurrent neural networks with time-varying delays," IEEE Transactions on Circuits and Systems I, vol. 50, no. 10, pp. 1353-1358, 2003.

[6] J. Cao and J. Wang, "Global asymptotic stability of a general class of recurrent neural networks with time-varying delays," IEEE Transactions on Circuits and Systems I, vol. 50, no. 1, pp. 34-44, 2003.

[7] K. Gopalsamy, "Stability of artificial neural networks with impulses," Applied Mathematics and Computation, vol. 154, no. 3, pp. 783-813, 2004.

[8] M. Jiang, Y. Shen, and X. Liao, "Global stability of periodic solution for bidirectional associative memory neural networks with varying-time delay," Applied Mathematics and Computation, vol. 182, no. 1, pp. 509-520, 2006.

[9] Y. Li, W. Xing, and L. Lu, "Existence and global exponential stability of periodic solution of a class of neural networks with impulses," Chaos, Solitons and Fractals, vol. 27, no. 2, pp. 437-445, 2006.

[10] B. Liu and L. Huang, "Existence and exponential stability of almost periodic solutions for cellular neural networks with mixed delays," Chaos, Solitons and Fractals, vol. 32, no. 1, pp. 95-103, 2007.

[11] Z. Liu and L. Liao, "Existence and global exponential stability of periodic solution of cellular neural networks with time-varying delays," Journal of Mathematical Analysis and Applications, vol. 290, no. 1, pp. 247-262, 2004.

[12] L. Zhou and G. Hu, "Global exponential periodicity and stability of cellular neural networks with variable and distributed delays," Applied Mathematics and Computation, vol. 195, no. 2, pp. 402-411, 2008.

[13] R. E. Gains and J. L. Mawhin, Coincidence Degree and Nonlinear Dfferential Equations, Springer, Berlin, Germany, 1977. 
[14] H. Wu, Y. Xia, and M. Lin, "Existence of positive periodic solution of mutualism system with several delays," Chaos, Solitons and Fractals, vol. 36, no. 2, pp. 487-493, 2008.

[15] A. Berman and R. J. Plemmons, Nonnegative Matrices in the Mathematical Sciences, Computer Science and Applied Mathematics, Academic Press, New York, NY, USA, 1979.

[16] J. P. LaSalle, The Stability of Dynamical Systems, SIAM, Philadelphia, Pa, USA, 1976. 


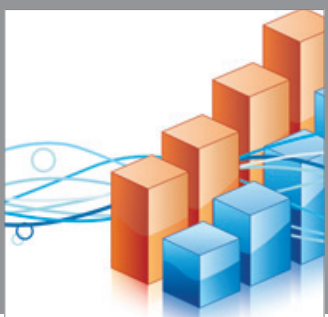

Advances in

Operations Research

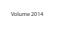

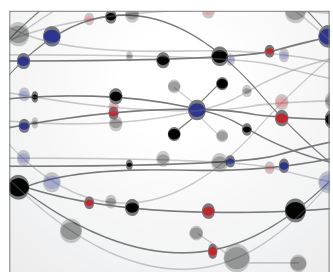

\section{The Scientific} World Journal
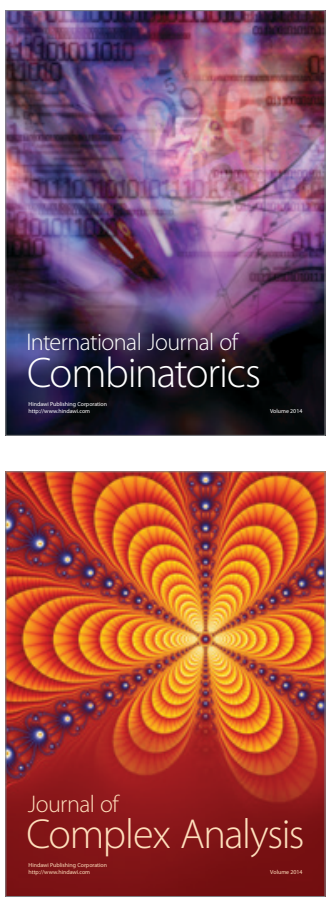

International Journal of

Mathematics and

Mathematical

Sciences
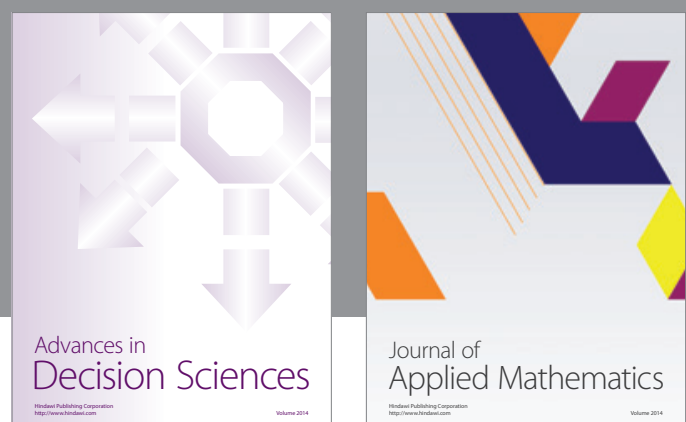

Journal of

Applied Mathematics
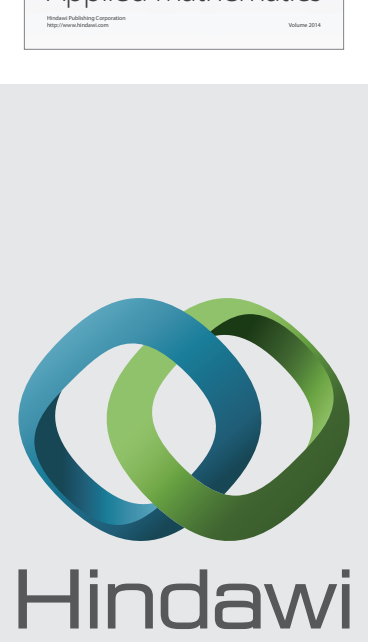

Submit your manuscripts at http://www.hindawi.com
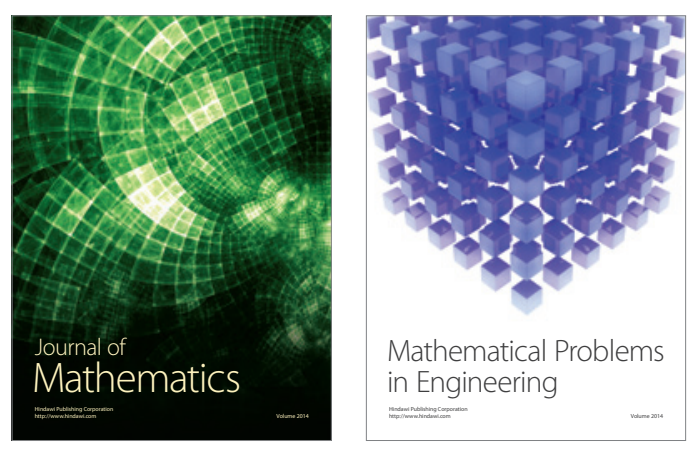

Mathematical Problems in Engineering
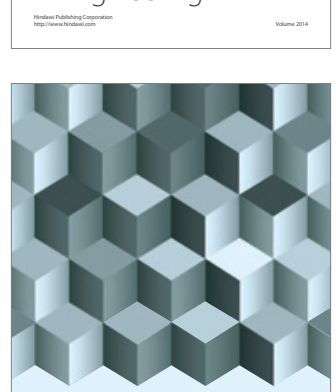

Journal of

Function Spaces
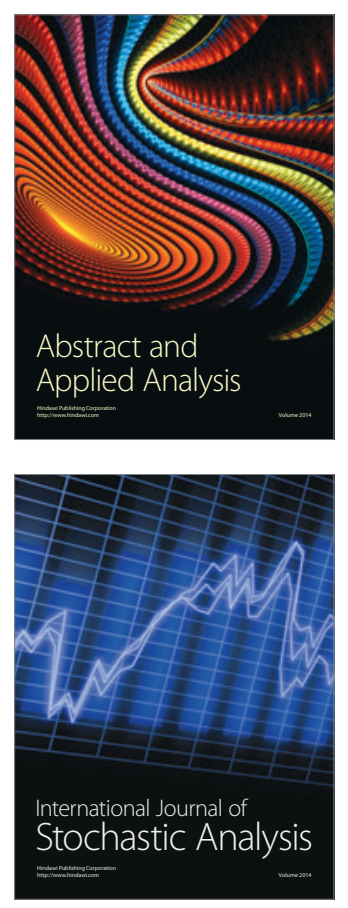

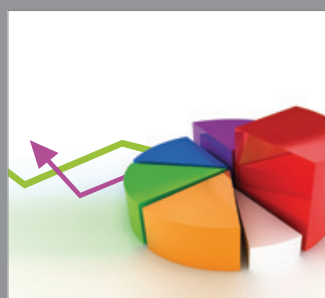

ournal of

Probability and Statistics

Promensencen
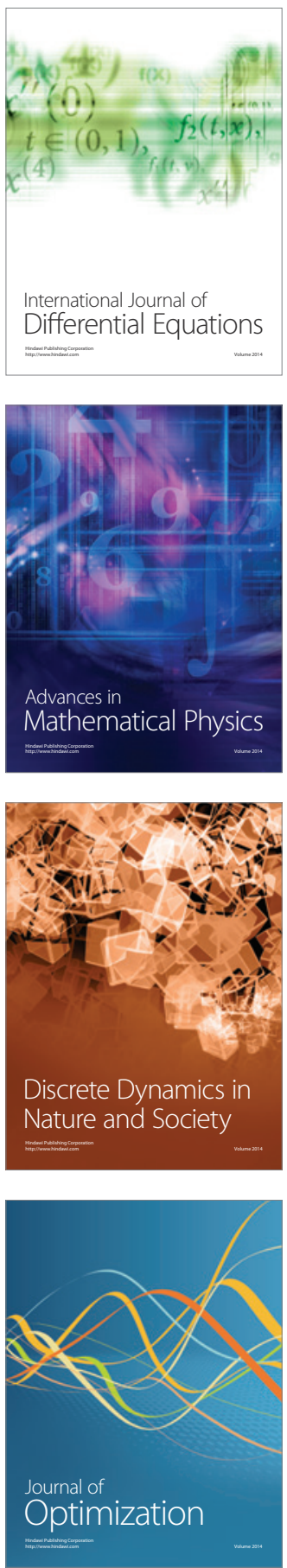